\title{
Safety and Efficacy of Gliflozin Group Among Patients with Type 2 Diabetes Including Patient's Satisfaction in Saudi Arabia
}

\author{
Al Hejji $R^{1 *}$, Al Ghamdi $M^{1}$, Al Haqbani $D^{1}$, Al Anazi B ${ }^{1}$, Al Bajjal $S^{1}$, Al Ghadeer $A^{1}$, Al Najjar $A^{2}$ and Al \\ Suwaidan $\mathbf{S}^{3}$ \\ ${ }^{1}$ College of Pharmacy, Riyadh, KSA \\ ${ }^{2} B$ Pharm, MsFS TQM, Drug \& Poison Information Supervisor, SFH, Riyadh, KSA
}

${ }^{3}$ Research Consultant, Health Sciences Research Center, PNU, Riyadh, KSA

*Corresponding author: Al Hejji R, College of Pharmacy, Riyadh, KSA

\section{ARTICLE INFO}

Received: 幽 May 25, 2019

Published: 幽 June 03, 2019

Citation: Al Hejji R, Al Ghamdi M, Al Haqbani D, Al Anazi B, Al Bajjal S, et al. Safety and Efficacy of Gliflozin Group Among Patients with Type 2 Diabetes Including Patient's Satisfaction in Saudi Arabia. Biomed J Sci \& Tech Res 18(4)2019. BJSTR. MS.ID.003173.

Keywords: SGLT2 inhibitors;T2DM; Fasting; Thirst; Tolerance

Abbreviations: SGLT2: SodiumGlucose Co-Transporter 2; T2DM: Type 2 Diabetes Mellitus; IRB: Institutional Review Board; PNU: Princess Nourah University; FBG: Fasting Blood Glucose; BMI: Body Mass Index; UTI: Urinary Tract Infections; GLP: Glucagon-like Peptide; ARB: Angiotensin Receptor Blockers; ACEI: Angiotensin Converting Enzyme Inhibitor; BB: Beta Blockers; CCB: Calcium Channel Blockers

\section{ABSTRACT}

Introduction: Sodium-Glucose Co-transporter 2 (SGLT2) inhibitors is the newest class of oral hypoglycemic agents that was approved for the management of patients with T2DM. Gliflozin group (Empagliflozin, Dapagliflozin \& Canagliflozin) have a unique mechanism of action in reducing hyperglycemia by decreasing renal glucose reabsorption and promoting urinary glucose excretion. The aim of this research is to monitor and evaluate the clinical efficacy and safety of (SGLT2) inhibitors for the treatment of type 2 diabetes.

Methodology: This study is a prospective cohort study supported by a questionnaire conducted by patients' interview. Patients' lab results were taken at baseline and 3-6 months later. This study, also, compared between two groups of patients; (26 patients) on Dapagliflozin and (23 patients) on Empagliflozin to compare the difference between their effects on HbA1c, FBG, Weight, BMI, LDL, HDL, TG, and total cholesterol.

Result: Treatment with gliflozin group resulted in a significant reduction on $\mathrm{HbA1c}$ by (1\%), FBG by $(2.77 \mathrm{mmol} / \mathrm{l})$ and BMI by $\left(0.56 \mathrm{~kg} / \mathrm{m}^{2}\right)$ over 3 to 6 months. On the other hand, there was no significant difference in the comparison between Dapagliflozin and Empagliflozin group. During Ramadan month $(46.2 \%)$ of the patients were very satisfied by the effect of gliflozin group, furthermore, no serious side effects were reported this month.

Conclusion: Gliflozin group was generally well tolerated, no serious side effect or hypoglycemic events have been reported during or after Ramadan. Empagliflozin have a favorable effect in reducing HbA1c and FBG over Dapagliflozin. However, Dapagliflozin has a better influence on weight reduction.

\section{Summary}

Subject of this study are susceptible to hypoglycemia especially in Ramadan month or fasting days. Also, the rate of night urination in this population is higher which may make them more liable to volume depletion. Additionally, type 2 diabetes patients need to manage their weight to maintain a good glucose control. Compliance and tolerance are always a challenge in patients with chronic disease.
Gliflozin group have the following characteristics:

a. Less hypoglycemic side effect

b. A positive effect on weight reduction

c. Help in controlling the glucose level

d. Tolerable with no serious side effects 
Empagliflozin have a favor effect in reducing $\mathrm{HbA1c}$ and $\mathrm{FBG}$ over Dapagliflozin. However, Dapagliflozin have a better influence on weight reduction in comparison to Empagliflozin. During Ramadan month $(46.2 \%)$ of the patients were very satisfied from the effect of gliflozin group. Due to the unique properties of gliflozin group in weight reduction and low risk of hypoglycemia, all these findings make gliflozin group a better choice for obese patients and who are at risk of hypoglycemia. Therefore, in comparison to other hypoglycemic agents as sulfonylureas and insulin therapy, which induce hypoglycemia and weight gain, consider the best option in diabetes treatment.

\section{Introduction}

Type 2 diabetes is a disease affecting metabolic disorders characterized by chronic hyperglycemia. Type 2 diabetes (T2DM) is resulting from either resistance to insulin action, inadequate insulin secretion, excessive or inappropriate glucagon secretion or a combination of two or more influences [1]. Prevalence of diabetes mellitus in Saudi Arabia is $17.7 \%$ and $12-14 \%$ with impaired glucose intolerance. While type 2 diabetes is $12.1 \%$, most of the patients on oral hypoglycemics [2-4]. The first part of the body that has been affected by type 2 diabetes is $\beta$-cell function which declines as a result to insulin resistance or reduces the insulin release. This will affect body metabolism leading to the challenge on how to maintain glucose level consistency [5,6]. Sodium-Glucose Co-transporter 2 (SGLT2) inhibitors is the newest class of oral hypoglycemic agents approved for the management of patients with type 2 diabetes mellitus (T2DM). Empagliflozin (Jardiance), dapagliflozin (Farxiga) and Canagliflozin (Invokana) are SGLT2 inhibitors that have recently been approved by regulation authorities in Saudi Arabia to treat T2DM [7].

Empagliflozin (10 mg, $25 \mathrm{mg}$ ), Dapagliflozin (5 mg, $10 \mathrm{mg}$ ) \& Canagliflozin $(100 \mathrm{mg}, 300 \mathrm{mg}$ ) were approved by FDA (Aug 1, 2014), (Jan 8, 2014) \& (Mar 29, 2013), respectively $[8,9]$. Gliflozin group have a unique mechanism of action in reducing hyperglycemia by decreasing renal glucose reabsorption and promoting urinary glucose excretion [10]. Unlike the other oral hypoglycemic agents from several other classes, the efficacy of gliflozin group is independent on insulin secretion and action. Therefore, when used in combination with other hypoglycemic agents, gliflozin group provides complementary therapy. In addition to weight loss and moderate reduction in blood pressure [11]. Gliflozins (Empagliflozin \& Canagliflozin) reduce the risk of the cardiovascular death, nonfatal myocardial infarction and nonfatal stroke [12]. The most reported adverse effects that could be associated with gliflozins are urinary tract infection, genital mycotic infections (particularly in women), increase urination, and upper respiratory tract infection $[13,14]$

The safety consideration and tolerability of gliflozin group are rare hypoglycemia, weight reduction, BP reduction, and is effective at all stages of T2DM. The risk observed was genitourinary infections, polyuria, volume depletion, hypotension, dizziness and increase in LDL-C, furthermore it is not recommended in patients with moderate or severe renal impairment [7]. Primary end point is to monitor and evaluate the clinical efficacy and safety of gliflozin group with overviewing the pattern and quality of use in the treatment of T2DM. Secondary end point is to compare the utilization of dapagliflozin compared to empagliflozin in patients with T2DM and their tolerability during Ramadan.

\section{Methodology and Clinical Study Design}

This study is a prospective cohort study supported by a questionnaire which conducted by patients' interview. Also, this study is to compare between two groups of patients, (26 patients) on Dapagliflozin and (23 patients) on Empagliflozin from the perspective of their effects on HbA1c, FBG, Weight, BMI, LDL, HDL, $\mathrm{TG}$ and total cholesterol.

\section{Ethics Approval}

The study was conducted according to Declaration of Helsinki. The study protocol was approved by Institutional Review Board (IRB) at Princess Nourah University (PNU) and Security Forces Hospital.

\section{Study Population}

A sample size of 71 patients' files were identified from diabetes clinics at Security Forces Hospital, Dallah Hospital and King Salman Hospital in Riyadh - Saudi Arabia in patients with T2DM. Out of them nineteen files were excluded because $n=2$ didn't have enough data and $n=17$ stopped the medication in less than two months. At the end 52 patients were included in the trial.

\section{Monitoring Variables}

The monitored variables in this study were: fasting blood glucose (FBG), HbA1c, weight, body mass index, lipid profile (LDL, HDL, TG), liver enzymes (AST, ALT) and serum creatinine. All the previous parameters were evaluated by reviewing patients' clinical files at baseline and three to six months after.

\section{Statistical Consideration}

Data were extracted from patients' profile and collected in an excel sheet to evaluate the efficacy of the medication. Then it was transferred to SPSS for statistical analysis. The data from questionnaires were also collected in another excel sheet and have been analyzed to find out patients' satisfaction and safety of the medication. Also, it was transferred to SPSS for analysis of the data using the frequency table.

\section{Result}

This study was carried on 71 patients receiving one type of gliflozin group. Then 19 patients were dropped due to not continuing their medications, to end up with 52 patients only enrolled in the study. These patients were on Dapagliflozin $(n=26)$, Empagliflozin $(n=23)$, and Canagliflozin $(n=3)$. The average of age 
was 49.75 ( \pm 10 SD), $63.5 \%$ were male and $36.5 \%$ were female. A readings. The baseline demographics and clinical characteristics duration of 3 to 6 months was between baseline readings and last are summarized in (Table 1).

Table 1: This table represents patient demographics data and characteristics at baseline.

\begin{tabular}{|c|c|c|c|}
\hline & Dapagliflozin (\%) & Empagliflozin (\%) & Canagliflozin (\%) \\
\hline No. study subjects & $\mathrm{n}=26(50 \%)$ & $\mathrm{n}=23(44 \%)$ & $\mathrm{n}=3(6 \%)$ \\
\hline Age mean & 48.5 & 51.57 & 46.67 \\
\hline \multicolumn{4}{|c|}{ Gender } \\
\hline Male (60\%) & 17 & 14 & 2 \\
\hline Female $(40 \%)$ & 9 & 9 & 1 \\
\hline \multicolumn{4}{|c|}{ Weight and body mass index (BMI) } \\
\hline Weight (kg) (SD) & $89.62(15.69)$ & $85.56(10.97)$ & $95.73(16)$ \\
\hline BMI (kg/m2) (SD) & $32.91(6)$ & $31.24(4.75)$ & $34.64(11.5)$ \\
\hline \multicolumn{4}{|c|}{ Concomitant medications received by study subjects } \\
\hline Insulin & 7 & 10 & 0 \\
\hline ACE inhibitors & 6 & 2 & 0 \\
\hline Diuretics & 4 & 5 & 0 \\
\hline Statin & 15 & 16 & 1 \\
\hline OG lowering agent & 25 & 20 & 3 \\
\hline
\end{tabular}

$\mathrm{OG}=$ oral glucose.

$\mathrm{ACE}=$ Angiotensin converting enzyme

\section{Patient Satisfaction}

A questionnaire has been developed (in house) to find out patient's satisfaction. This was conducted after three months from receiving one type of gliflozin group. All patients have signed the consent form. The objective of this questionnaire is to evaluate the tolerability of gliflozin group in type 2 diabetes during Ramadan month. Since Ramadan month (2018) came during hot weather, the study was focusing mainly on thirst, nocturia, feeling physically good \& fear of hypoglycemia, due to the hot weather and long day during "fasting month". It has been noticed that gliflozin group was generally well tolerated during Ramadan month and fasting days. The incidence of thirst was seen in $23.1 \%$ of the patients however, it doesn't consider very severe. All patients were suffering from nocturia were categorized depending on number of urinations per night into mild (1-3), moderate (4-6) and severe (7-10) where $(40.4 \%, 23.1 \%, 36.5 \%)$ respectively.

Nocturia occurred on patients especially in the beginning of Ramadan. Urination during the fasting days was decreased later where their body can tolerate fasting. Furthermore, out of the whole gliflozin group there were 7 patients $(13.5 \%)$ afraid from hypoglycemia, 4 patients $(7.7 \%)$ were interrupted their fasting because of hypoglycemia, and 4 patients (7.7\%) were stopped their medications for short period of time, then resume, due to fearing from side effects. On the other hand, $46.2 \%$ of the patients were very satisfied from the effect of gliflozin group. No urinary tract infections (UTI) or genital mycosis infection were reported with either empagliflozin, dapagliflozin or canagliflozin among patients during Ramadan month.

\section{Safety Profile}

As mentioned before, only 4 patients $(7.7 \%)$ who had their fasting interrupted, our results showed that there was no difference in total cholesterol, liver function test and serum creatinine before and after baseline for any of the gliflozin group. Mean reduction for total cholesterol was $0.4 \mathrm{mmol} / \mathrm{L}$ ( 4.91 at baseline and 4.56 after). It was also noticed a reduction in the mean of ALT and AST. The baselines were 32.04 (ALT), 19.87 (AST) and then the later readings were 29.48 (ALT), and 21.65 (AST). The mean serum creatinine at the baseline was 71.87 and 70.4 after. However, none of the reductions was significant $(\mathrm{P}>0.05)$. The safety of gliflozin group with concomitant medications represents that all types of gliflozin were safe with insulin, ACE inhibitors, diuretics, Statin and oral hypoglycemic agents. There were no side effects reported because of gliflozin group either as monotherapy or with concomitant medications, also there were no hypoglycemic events during Ramadan or after Ramadan. The frequently used medications are represented in (Figures 1 \& 2). 


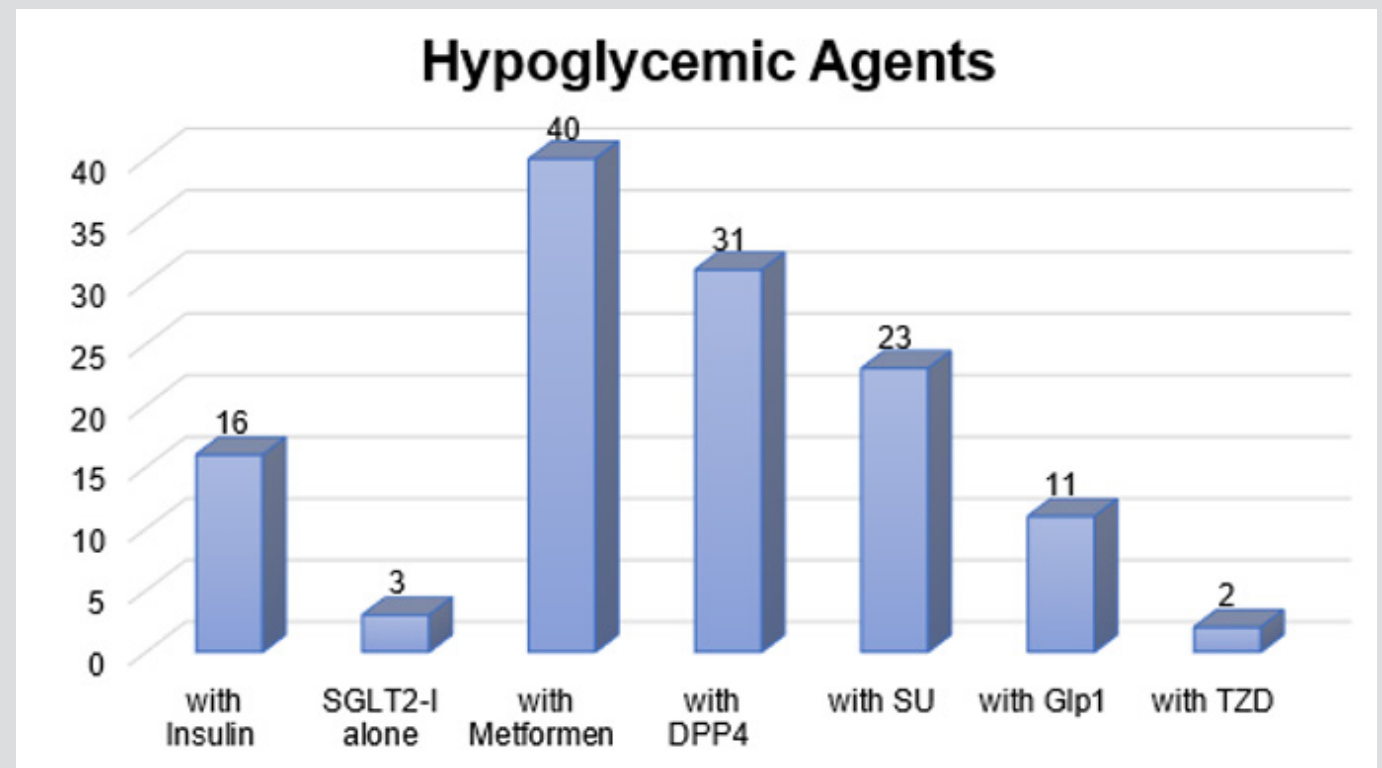

SGLT2-I: Sodium Glucose co-Transporter 2 Inhibitors, DPP4: Dipeptidyl peptidase-4 inhibitor, SU: Sulphonylureas, Glp1: Glucagon-like peptide-1, TZD: Thiazolidinedione.

Figure 1: This figure represents the frequently used number of oral hypoglycemics consumed by the population.

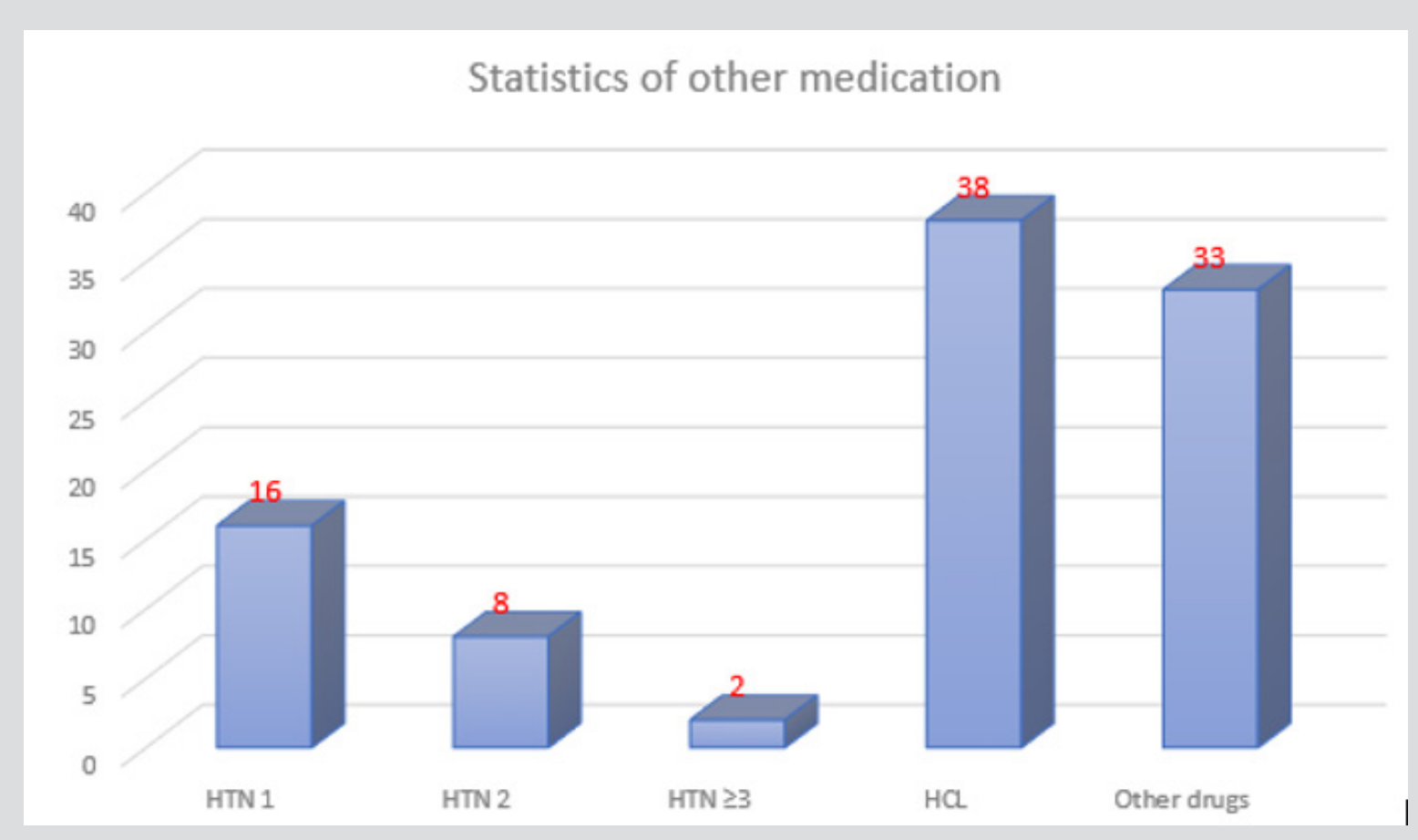

HTN1: using 1 class of hypotensive medication. HTN2: using a combination of 2 different classes of hypotensive medications. $\mathrm{HNT} \geq 3$ : using a combination of three or more type of hypotensive medications.

HCL: Hypercholesterolemia drugs.

Figure 2: Number of drug classes frequently used by the study population.

\section{Glycemic Efficacy}

In this study it has been noticed that gliflozin group reduced HbA1c level with almost 1\% (the average HbA1c at baseline readings was $8.85 \%$ and last reading was $7.89 \%$ ). This reduction of HbA1c was significantly different $(\mathrm{P}<0.001)$ showing that most of the patients had a reduction in their $\mathrm{HbA1c}$. Similar results were found in fasting blood glucose (FBG) reduction in the last readings with a significant difference $(\mathrm{p}<0.001)$. The reduction of FBG was decreased from $12 \mathrm{mmol} / \mathrm{L}$ to $9.23 \mathrm{mmol} / \mathrm{L}$. 


\section{Body Mass Index (BMI) Efficacy}

Results revealed a major difference between baseline and last readings for the BMI with means $\left(32.28,31.72 \mathrm{~kg} / \mathrm{m}^{2}\right)$ respectively.
The reduction in BMI was $\left(0.56 \mathrm{~kg} / \mathrm{m}^{2}\right)$, showing statistically significant $(\mathrm{P}<0.05)$. Table 2 illustrated more detailed information.

Table 2: This table represents the mean and standard deviation of lab variables before and after taking SGLT-2 inhibitors.

\begin{tabular}{|c|c|c|c|c|c|c|}
\hline \multirow[b]{2}{*}{ Variable } & \multicolumn{2}{|c|}{ Before } & \multicolumn{2}{|c|}{ After } & \multirow[t]{2}{*}{ Difference Average } & \multirow[t]{2}{*}{ P-value } \\
\hline & Mean & STD & Mean & STD & & \\
\hline BMI & 32.28 & 5.86 & 31.72 & 5.17 & 0.56 & 0.00488 \\
\hline FBG & 12 & 3.83 & 9.23 & 3.24 & 2.77 & 0.00007 \\
\hline $\mathrm{HbA1c}$ & 8.85 & 2.03 & 7.89 & 1.76 & 0.96 & 0.00009 \\
\hline LDL & 2.26 & 1.82 & 2.78 & 1.55 & -0.52 & 0.05916 \\
\hline HDL & 1.03 & 0.28 & 1.11 & 0.38 & -0.07 & 0.1016 \\
\hline TG & 3.8 & 7.48 & 2.18 & 1.39 & 1.62 & 0.13293 \\
\hline Total Chol. & 4.91 & 2.38 & 4.56 & 1.18 & 0.36 & 0.28177 \\
\hline AST & 19.87 & 9.97 & 21.65 & 11.97 & -1.78 & 0.44875 \\
\hline ALT & 32.04 & 20.12 & 29.48 & 16 & 2.56 & 0.17818 \\
\hline $\mathrm{SCr}$ & 71.87 & 18.48 & 70.4 & 19.5 & 1.47 & 0.77504 \\
\hline Bilirubin & 7.87 & 3.14 & 9.3 & 5.95 & -1.43 & 0.29478 \\
\hline ALK. Phos. & 78.92 & 25.05 & 76.29 & 24.66 & 2.63 & 0.06486 \\
\hline Albumin & 43.08 & 3.48 & 43.7 & 3.4 & -0.62 & 0.13477 \\
\hline
\end{tabular}

\section{Dapagliflozin Vs. Empagliflozin}

In comparison between Dapagliflozin and Empagliflozin groups, it has been found that Dapagliflozin have a better influence in reducing the weight and body mass index (BMI) than Empagliflozin, the mean $( \pm \mathrm{SD})$ weight reduction is $(2.34 \mathrm{Kg} \pm 3.7,0.41 \mathrm{Kg} \pm 3.7)$ respectively, however this reduction was insignificant $(\mathrm{P}>0.05)$. Furthermore, Empagliflozin have a favor effect in reducing $\mathrm{HbA1c}$ and FBG over Dapagliflozin. The mean reduction was $3.45 \mathrm{mmol} / \mathrm{L}$ \pm 2.9 (FBG), $1.29 \% \pm 2.13(\mathrm{HbA} 1 \mathrm{c})$ for Empagliflozin and 1.81 $\mathrm{mmol} / \mathrm{L} \pm 1.86$ (FBG), $0.72 \% \pm 1.01$ (HbA1c) for Dapagliflozin, hence this reduction was insignificant $(\mathrm{P}>0.05)$. Additionally, all other tests including liver enzymes and cholesterol levels have similar results with no significant differences. Table 3 summarizes the differences between Dapagliflozin \& Empagliflozin (Table 3).

Table 3: This table represents the difference between Dapagliflozin and Empagliflozin with the mean of adjusted value.

\begin{tabular}{|c|c|c|c|c|c|}
\hline \multirow[b]{2}{*}{ Variable } & \multicolumn{2}{|c|}{ Dapagliflozin } & \multicolumn{2}{|c|}{ Empagliflozin } & \multirow[b]{2}{*}{ Difference Average } \\
\hline & Mean & STD & Mean & STD & \\
\hline Weight & 2.3423 & 3.7177 & 0.4091 & 3.7497 & 1.9332 \\
\hline BMI & 0.9323 & 1.6035 & 0.2117 & 1.3799 & 0.7206 \\
\hline FBG & 1.805 & 1.8618 & 3.4488 & 2.898 & -1.6438 \\
\hline $\mathrm{HbA1c}$ & 0.7192 & 1.0147 & 1.2896 & 2.1269 & -0.5703 \\
\hline LDL & 0.0195 & 0.6908 & -0.6982 & 2.1158 & 0.7177 \\
\hline HDL & -0.0325 & 0.1927 & -0.0558 & 0.2014 & 0.0233 \\
\hline TG & 0.5225 & 1.385 & 3.0835 & 10.7405 & -2.561 \\
\hline Total Chol. & 0.125 & 0.7535 & 0.6004 & 3.3331 & -0.4754 \\
\hline
\end{tabular}

\section{Discussion}

This study is prospective, observational conducted to evaluate the clinical efficacy and safety of gliflozin group as being utilized for type 2 diabetes alone or in combination with other oral hypoglycemic agents. It is also to compare the utilization of dapagliflozin with empagliflozin for the treatment of type 2 diabetes. In addition, it focused on patient satisfaction to evaluate the physical and emotional effect of the gliflozin group on these patients. As mentioned earlier, the mechanism of action of this group is by inhibiting sodium glucose co-transporter 2 (SGLT-2), which facilitates renal reabsorption of glucose $[15,16]$. The glycemic efficacy for gliflozin group has been assessed to reduce HbA1c by $1 \%[8,17,18]$. Where this study has similar results to reduce HbA1c level with almost $1 \%$. It is also found that $\mathrm{HbA1c}$ reduction was higher with empagliflozin compared to dapagliflozin although the difference was not statistically significant.

There was a very low incidence rate of hypoglycemic episodes, where most of the patients didn't interrupt their fasting. 
Furthermore, patients who suffered from nocturia at the first days of Ramadan were categorized as mild (1-3), moderate (4-6) and severe $(\geq 7)$ according to the number of urinations per night indicated by the patients in the questionnaire. The results showed that $40.4 \%$ of the patients have mild nocturia and overall tolerable. Neither urinary tract infections (UTI) nor genital infection were observed in the study subjects. The effect of thirst was very slight upon a patient who fasted approximately 15 hours a day; this action makes them very satisfied. In general, gliflozin group have a good safety profile with tolerable side effects. The most frequent side effect during fasting month was nocturia and thirst. Some studies assessed the safety of SGLT-2 inhibitors during fasting month and they found a similar result $[19,20]$. Gliflozin group induced insignificant changes in lipid profile, small increase in HDL-C, LDL-C and minor reduction in triglyceride levels. These findings were similar to [21,22] where the ratio of LDL/HDL cholesterol remains constant.

Empagliflozin and Dapagliflozin provide a sustained glycemic and weight control and were well tolerated with a low risk of hypoglycemia in patients with type 2 diabetes. T2DM is associated with obesity, and especially visceral/abdominal adiposity, insulin resistance, metabolic syndrome, and increased cardiovascular risk $[23,24]$. Hypoglycemic agents have the potential for increasing the weight such as: Sulfonylureas, Insulin, and Thiazolidinediones, some of them are weight neutral such as: Metformin, $\alpha$-Glucosidase inhibitors and Dipeptidyl peptidase- 4 inhibitors, and only a few agents have the effect of weight reduction such as: Glucagon-like peptide (GLP)-1 receptor agonist and SGLT-2 inhibitors [7,25,26]. This study shows that, Dapagliflozin have a better influence on weight reduction in comparison to Empagliflozin. The average difference between them was (1.9 kg). Empagliflozin, on the other hand used as monotherapy or add-on to Metformin to investigate the long-term safety and efficacy, showed clinically meaningful reductions in $\mathrm{HbA1c}$, FBG, body weight, and waist circumference compared with baseline. Moreover, it is associated with small reduction in uric acid and blood pressure $[27,28]$.

Concomitant use of two or more of oral hypoglycemic medications might increase the risk of hypoglycemia. Also, gliflozin group causes osmotic diuresis so, patients who take combination of gliflozin with diuretics may have a higher risk of appearing dehydration and hypotension as side effects [29]. On the other side, there is a hypothesis said that gliflozin can be used as an option in case of loop diuretic resistance in patient with heart failure to overcome the resistance [30]. However, patients who take these combinations should be monitored closely to avoid these side effects. There is no drug interaction found in this study for patient taking combination of gliflozin and statin for dyslipidemia or angiotensin receptor blockers (ARB), angiotensin converting enzyme inhibitor (ACEI), beta blockers (BB), calcium channel blockers (CCB), levothyroxine for hyperthyroidism and Vitamin B complex.

\section{Conclusion}

In summary, gliflozin group have a significant reduction in HbA1c level with almost $1 \%$ besides favorable significant weight reduction. Empagliflozin have a favor effect in reducing HbA1c and FBG over Dapagliflozin. However, Dapagliflozin have a better influence on weight reduction in comparison to Empagliflozin. In terms of safety, gliflozin group were generally well tolerated in Ramadan month and fasting days especially in hot weather. During Ramadan month $(46.2 \%)$ of the patients were very satisfied from the effect of gliflozin group. No serious side effects or hypoglycemic events have been reported.

\section{Acknowledgment}

The authors would like to thank Dr. Saher Safarini (Head of diabetic center in Dallah hospital), Dr. Eman sheshah (Head of diabetic center in king Salman hospital), Dr. Fahad Al-Sabaan (Head of Diabetic Care Unit, SFHP-Riyadh), Dr. Mayasa Qurashi, Consultant Endocrinologist, SFHP-Riyadh. This article is not funded.

\section{References}

1. Kohei KAKU (2010) Pathophysiology of Type 2 Diabetes and Its Treatment Policy. JMAJ 53(1): 41-46.

2. Alotaibi A, Perry L, Gholizadeh L, Al Ganmi A (2017) Incidence and prevalence rates of diabetes mellitus in Saudi Arabia: An overview. J Epidemiol Glob Health 7(4): 211-218.

3. Bahijri SM, Jambi HA, Al Raddadi RM, Ferns G, Tuomilehto J (2016) The Prevalence of Diabetes and Prediabetes in the Adult Population of Jeddah, Saudi Arabia - A Community-Based Survey. PLoS ONE 11(4): e0152559.

4. (2017) International Diabetes Federation, DIABETES ATLAS- Eighth edition. ISBN: 978-2-930229-87-4.

5. Nathan DM, Buse JB, Davidson MB, Ferrannini E, Holman RR (2009) Medical management of hyperglycemia in type 2 diabetes: a consensus algorithm for the initiation and adjustment of therapy: a consensus statement of the American Diabetes Association and the European Association for the Study of Diabetes. Diabetes Care 32(1): 193-203.

6. Bailey CG, Gross JL, Hennicken D, Iqbal N, Mansfield TA, et al. (2013) Dapagliflozin add-on to metformin in type 2 diabetes inadequately controlled with metformin: a randomized, double-blind, placebocontrolled 102-week trial. BMC Medicine 11: 43.

7. Matthew C, Bakris G, Blonde L, Andrew JM Boulton, David DAlessi, et al. (2018) Standards of Medical Care in Diabetes. Diabetes Care 41(Suppl 1): S73-S85

8. Mosley JF, Smith L, Everton E, Fellner C (2015) Sodium-Glucose Linked Transporter 2 (SGLT2) Inhibitors in the Management Of Type-2 Diabetes: A Drug Class Overview. P T 40(7): 451-462.

9. (2014) Product Information: INVOKANA(TM) oral tablets, canagliflozin oral tablets. Janssen Pharmaceuticals, Titusville, NJ.

10. Michael JB van Baar, Charlotte C (2018) SGLT2 Inhibitors in Combination Therapy: From Mechanisms to Clinical Considerations in Type 2 Diabetes Management. Diabetes Care 41(8): 1543-1556.

11. Nauck MA (2014) Update on developments with SGLT2 inhibitors in the management of type 2 diabetes. Drug Des Devel Ther 8: 1335-1380.

12. Rastogi A, Bhansali A (2017) SGLT2 Inhibitors Through the Windows of EMPA-REG and CANVAS Trials: A Review. Diabetes Ther 8(6): 12451251. 
13. Verma S, Mazer C ,Fitchett D, Inzucchi SE, Pfarr E, et al. (2018) Empagliflozin reduces cardiovascular events, mortality and renal events in participants with type 2 diabetes after coronary artery bypass graft surgery: subanalysis of the EMPA-REG OUTCOME ${ }^{\circledR}$ randomised trial. Diabetologia 61(8): 1712-1723.

14. Plosker GL (2012) Dapagliflozin: a review of its use in type 2 diabetes mellitus. Drugs 72(17): 2289-2312.

15. Riddle MC, Cefalu WT (2018) SGLT Inhibitors for Type 1 Diabetes: An Obvious Choice or Too Good to Be True. Diabetes Care 41(12): 24442447.

16. Bailey CJ (2011) Renal glucose reabsorption inhibitors to treat diabetes. Trends in Pharmacological Sciences 32(2): 63-71.

17. Schernthaner G, Gross JL, Rosenstock J, Guarisco M, Fu M, et al. (2013) Canagliflozin compared with sitagliptin for patients with type 2 diabetes who do not have adequate glycemic control with metformin plus sulfonylurea: A 52-week randomized trial. Diabetes Care 36(9): 25082515 .

18. Alaaeldin B, Khalifa A, Rashid F, Abdelgadir EI, Al Qaysi AA, et al. (2017) Efficacy and Safety of SGLT2 Inhibitors in ReducingGlycated Hemoglobin and Weight in Emirati Patients With Type 2 Diabetes. J Clin Med Res 9(6): 499-507.

19. Alaaeldin B, Khalifa A, Abdulgadir E, Al Saeed MA, Al Qaysi AA, et al. (2018) Safety of Sodium-Glucose Cotransporter 2 Inhibitors (SGLT2-I) During the Month of Ramadan in Muslim Patients with Type 2 Diabetes. Oman Med J 33(2): 104-110.

20. Seman W WJ, Kori, Rajoo S, Othman H, Mohd Noor N, et al. (2016) Switching from sulphonylurea to a sodium-glucose cotransporter 2 inhibitor in the fasting month of Ramadan is associated with a reduction in hypoglycemia, Diabetes. Obesity and Metabolism 18(6): 628-632.

21. Abdul Ghani M, Del Prato S, Chilton R, DeFronzo RA (2016) SGLT2 Inhibitors and Cardiovascular Risk: Lessons Learned From the EMPAREG OUTCOME Study. Diabetes Care 39(5): 717-725.

\section{ISSN: 2574-1241}

DOI: 10.26717/BJSTR.2019.18.003173

Al Hejji R. Biomed J Sci \& Tech Res

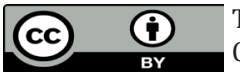

This work is licensed under Creative Commons Attribution 4.0 License

Submission Link: https://biomedres.us/submit-manuscript.php
22. Inzucchi SE, Zinman B, Wanner C, Ferrari R, Fitchett D, et al. (2015) SGLT2 inhibitors and cardiovascular risk: Proposed pathways and review of ongoing outcome trials. Diab Vasc Dis Res 12(2): 90-100.

23. Chen M, Xie CG, Gao H, Zheng H, Chen Q et al. (2016) Comparative effectiveness of sodiumglucose co-transporter 2 inhibitors for controlling hyperglycemia in patients with type 2 diabetes: protocol for a systematic review and network meta-analysis. BMJ Open 6(1): e010252.

24. List JF, Woo V, Morales E, Tang W, Fiedorek FT (2009) Sodium-Glucose Cotransport Inhibition With Dapagliflozin in Type 2 Diabetes. Diabetes Care 32(4): 650-657.

25. Inzucchi SE, Bergenstal RM, Buse JB, Diamant M, Ferrannini E, et al. (2015) Management of hyperglycemia in type 2 diabetes 2015: A patient-centeredapproach: Update to position statement of the American Diabetes Association and the European Associationfor the Study of Diabetes. Diabetes Care 38(1): 140-149.

26. Juang PS, Henry RR (2000) Treatment of type 2 diabetes. In: De Groot LJ, Beck-Peccoz P, Chrousos G, Dungan K, Grossman A, et al. (2015) Endotext [Internet]. South Dartmouth (MA): MDText.com.

27. Ferrannini E, Berk A, Hantel S, Sabine Pinnetti, Thomas Hach, et al (2013) Long-Term Safety and Efficacy of Empagliflozin, Sitagliptin, and Metformin. diabetes care 36(12): 4015-4402.

28. Inzucchi SE, Zinman B, Fitchett D, Wanner C, Ele Ferrannini, et al. (2018) How Does Empagliflozin Reduce Cardiovascular Mortality? Insights From a Mediation Analysis of the EMPA-REG OUTCOME Trial. Diabetes Care 41(2): 356-363.

29. (2018) BNF 76, Tavistock Square, London: BMJ Group pp. 1335-1337.

30. Wilcox CS, Shen W, Boulton DW, Leslie BR, Griffen SC (2018) Interaction Between the Sodium-Glucose-Linked Transporter 2 Inhibitor Dapagliflozin and the Loop Diuretic Bumetanide in Normal Human Subjects. J Am Heart Assoc 7(4): e007046. 